\title{
Enantioselective Synthesis of 3,3-Difluoropyrrolidin-4-ol, a Valuable Building Block in Medicinal Chemistry
}

Chong Si, ${ }^{*}{ }^{\dagger}$ Kevin R. Fales, ${ }^{\dagger}$ Alicia Torrado, ${ }^{\ddagger}$ Kwame Frimpong, ${ }^{\dagger}$ Talbi Kaoudi, ${ }^{\ddagger}$ Harold George Vandeveer, ${ }^{\S}$ F. George Njoroge ${ }^{\dagger}$

†DCR\&T, Lilly Research Laboratories, Eli Lilly and Company, Indianapolis, IN 46285, United States ${ }^{\ddagger}$ DCR\&T, Lilly Spain S.A., Avda de la Industria 30, Alcobendas- Madrid, 28108, Spain §AMRI Contractor to Eli Lilly in Indianapolis, IN, AMRI, 26 Corporate Circle, Albany, NY 12203

* Corresponding author. Tel.: +1-317-433-5853; e-mail: si_chong@lilly.com

\section{Table of Contents}

${ }^{1} \mathrm{H}$ and ${ }^{13} \mathrm{C}$ NMR Spectra of Compound 3

${ }^{1} \mathrm{H}$ and ${ }^{13} \mathrm{C}$ NMR Spectra of Compound 3a

${ }^{1} \mathrm{H},{ }^{19} \mathrm{~F}$ and ${ }^{13} \mathrm{C}$ NMR Spectra of Compound 4

Chiral HPLC analysis of Compound 4 S5

${ }^{1} \mathrm{H},{ }^{19} \mathrm{~F}$ and ${ }^{13} \mathrm{C}$ NMR Spectra of Compound $\mathbf{1}$

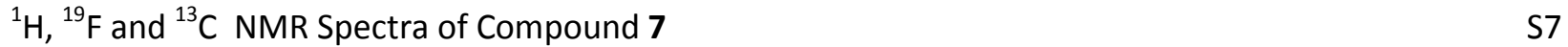

${ }^{1} \mathrm{H},{ }^{19} \mathrm{~F}$ and ${ }^{13} \mathrm{C}$ NMR Spectra of Compound 8

${ }^{1} \mathrm{H}$ and ${ }^{13} \mathrm{C}$ NMR Spectra of Compound $\mathbf{1 0}$ S9

${ }^{1} \mathrm{H},{ }^{19} \mathrm{~F}$ and ${ }^{13} \mathrm{C}$ NMR Spectra of Compound $\mathbf{1 1}$

${ }^{1} \mathrm{H},{ }^{19} \mathrm{~F}$ and ${ }^{13} \mathrm{C}$ NMR Spectra of Compound $\mathbf{1 2} \quad \mathrm{S} 11$

${ }^{1} \mathrm{H},{ }^{19} \mathrm{~F}$ and ${ }^{13} \mathrm{C}$ NMR Spectra of Compound $\mathbf{1 3} \quad$ S12

${ }^{1} \mathrm{H},{ }^{19} \mathrm{~F}$ and ${ }^{13} \mathrm{C}$ NMR Spectra of Compound $\mathbf{1 4} \quad$ S13

Chiral HPLC analysis of Compound $14 \quad$ S14

${ }^{1} \mathrm{H},{ }^{19} \mathrm{~F}$ and ${ }^{13} \mathrm{C}$ NMR Spectra of Compound 14a 


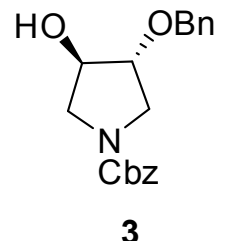

1H NMR, $\mathrm{CDCl}_{3}, 400 \mathrm{MHz}$

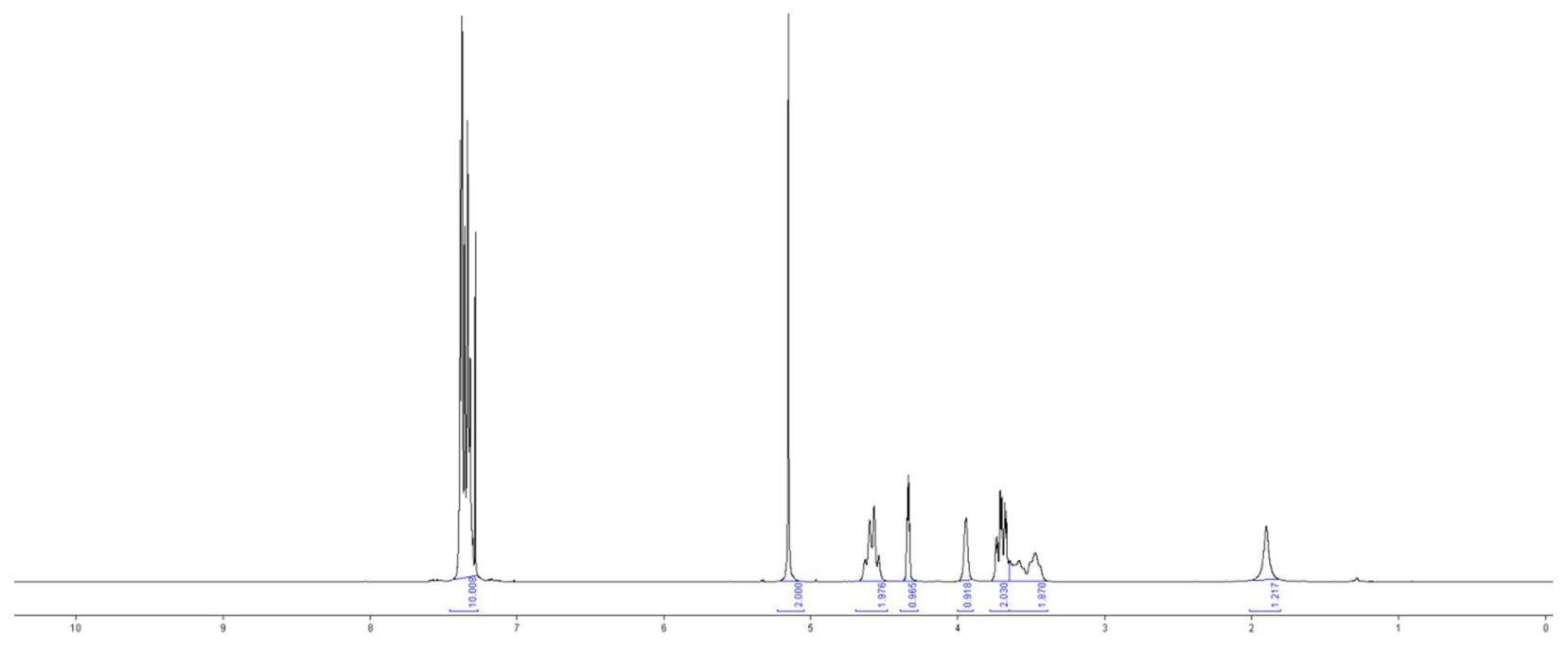

13C NMR, $\mathrm{CDCl}_{3}, 100 \mathrm{MHz}$

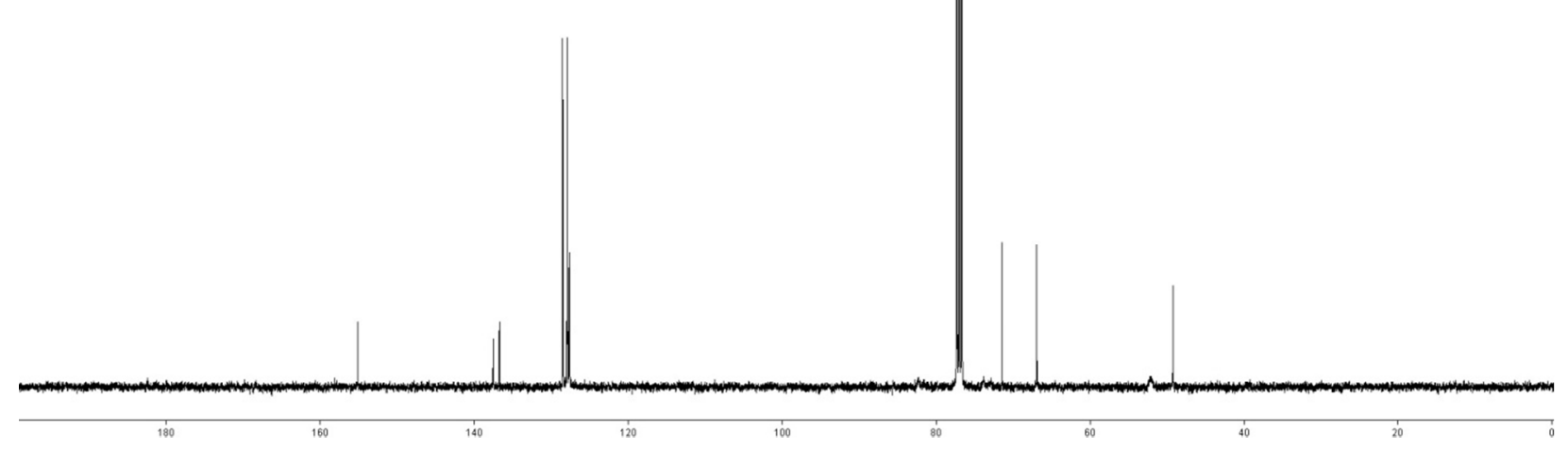




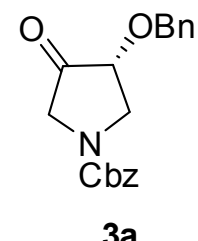

1H NMR, $\mathrm{CDCl}_{3}, 400 \mathrm{MHz}$

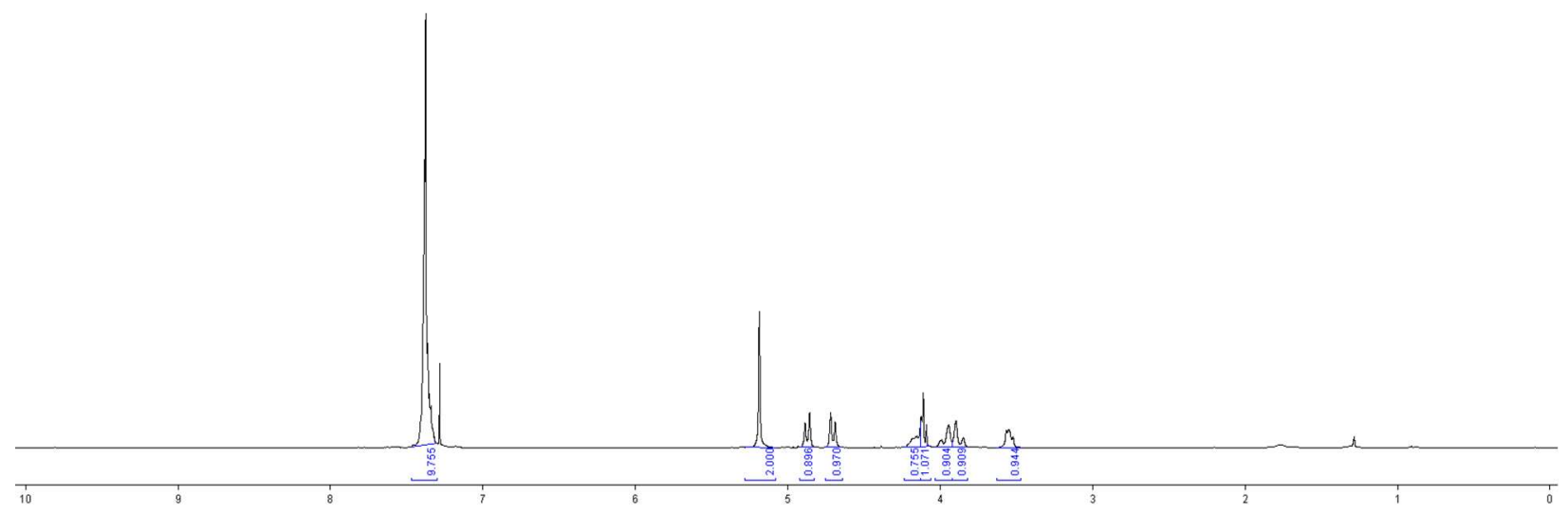

13C NMR, $\mathrm{CDCl}_{3}, 100 \mathrm{MHz}$

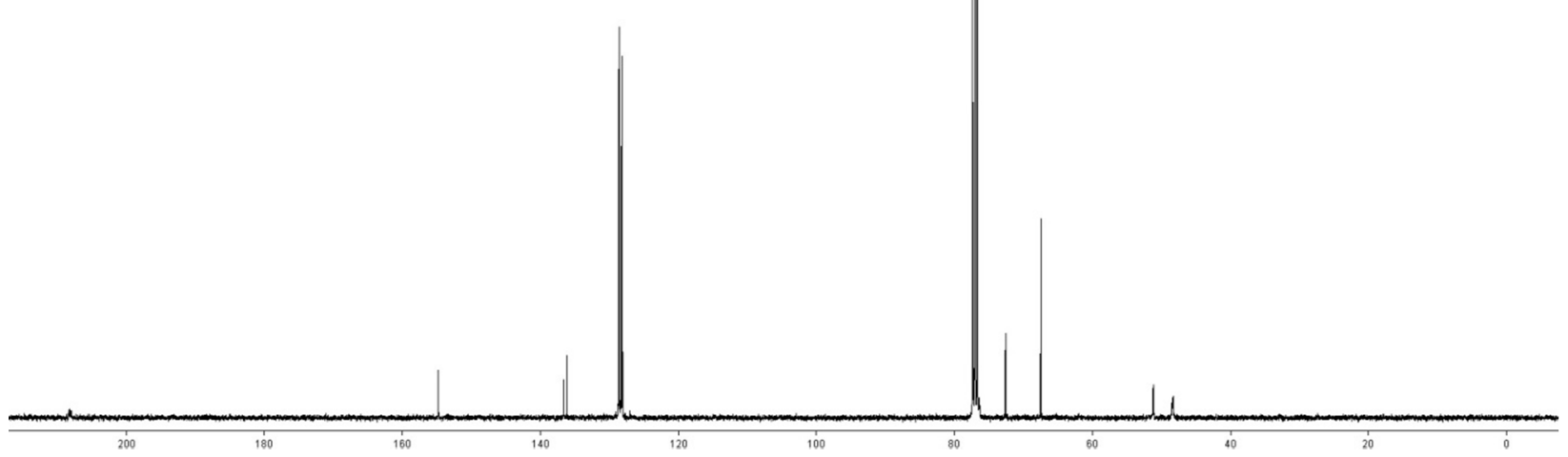




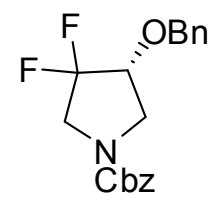

4

1H NMR, $\mathrm{CDCl}_{3}, 400 \mathrm{MHz}$

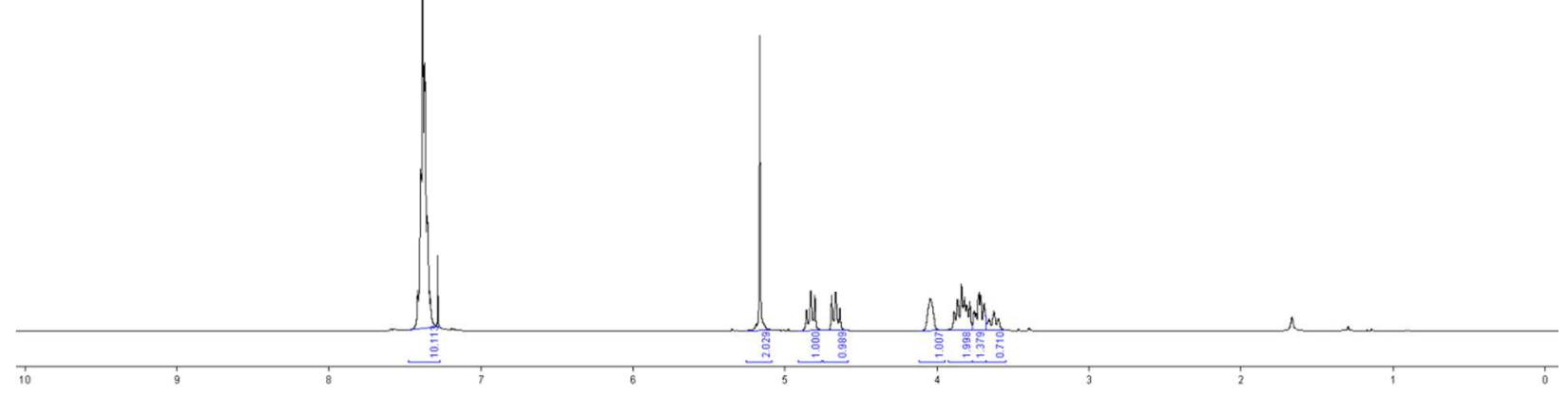

19F NMR, $\mathrm{CDCl}_{3}, 377 \mathrm{MHz}$

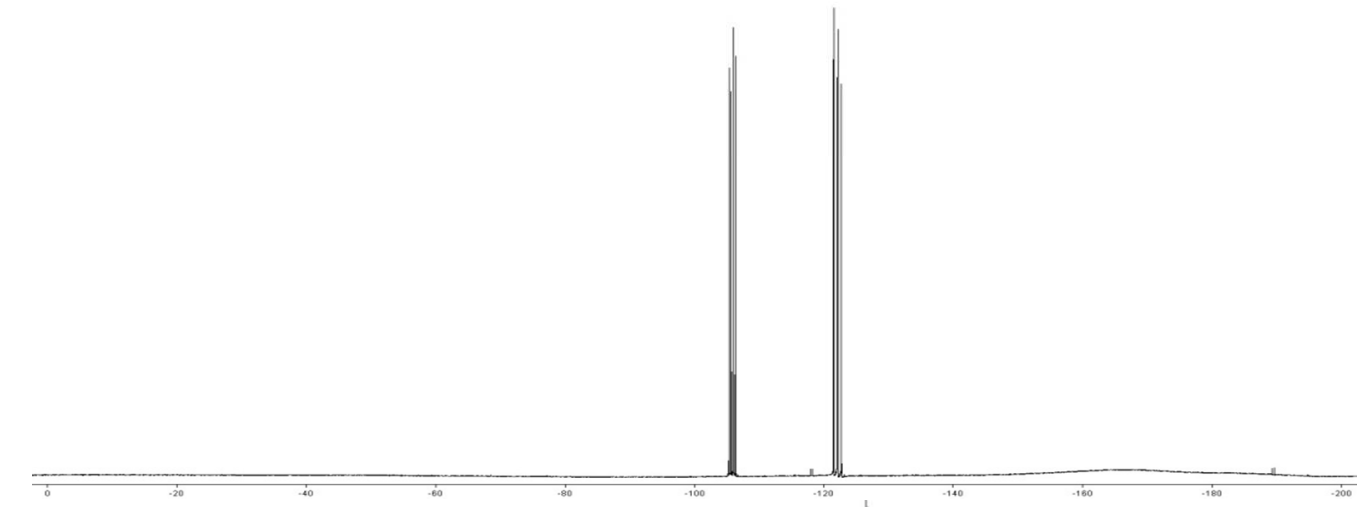

13C NMR, $\mathrm{CDCl}_{3}, 100 \mathrm{MHz}$

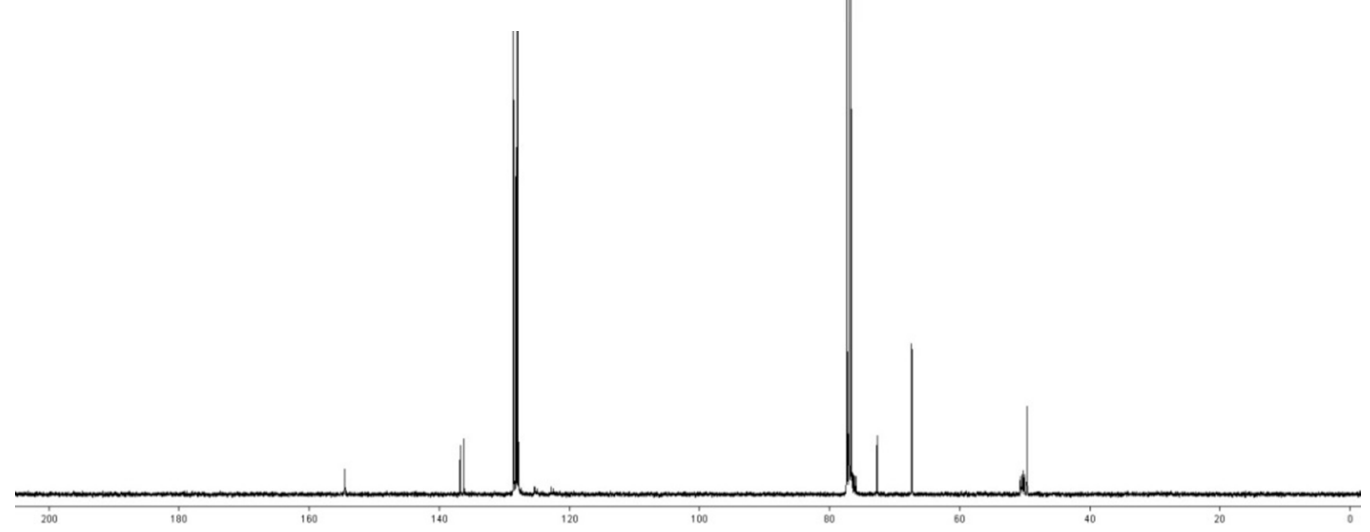




\section{Chiral HPLC analysis of compound 4}
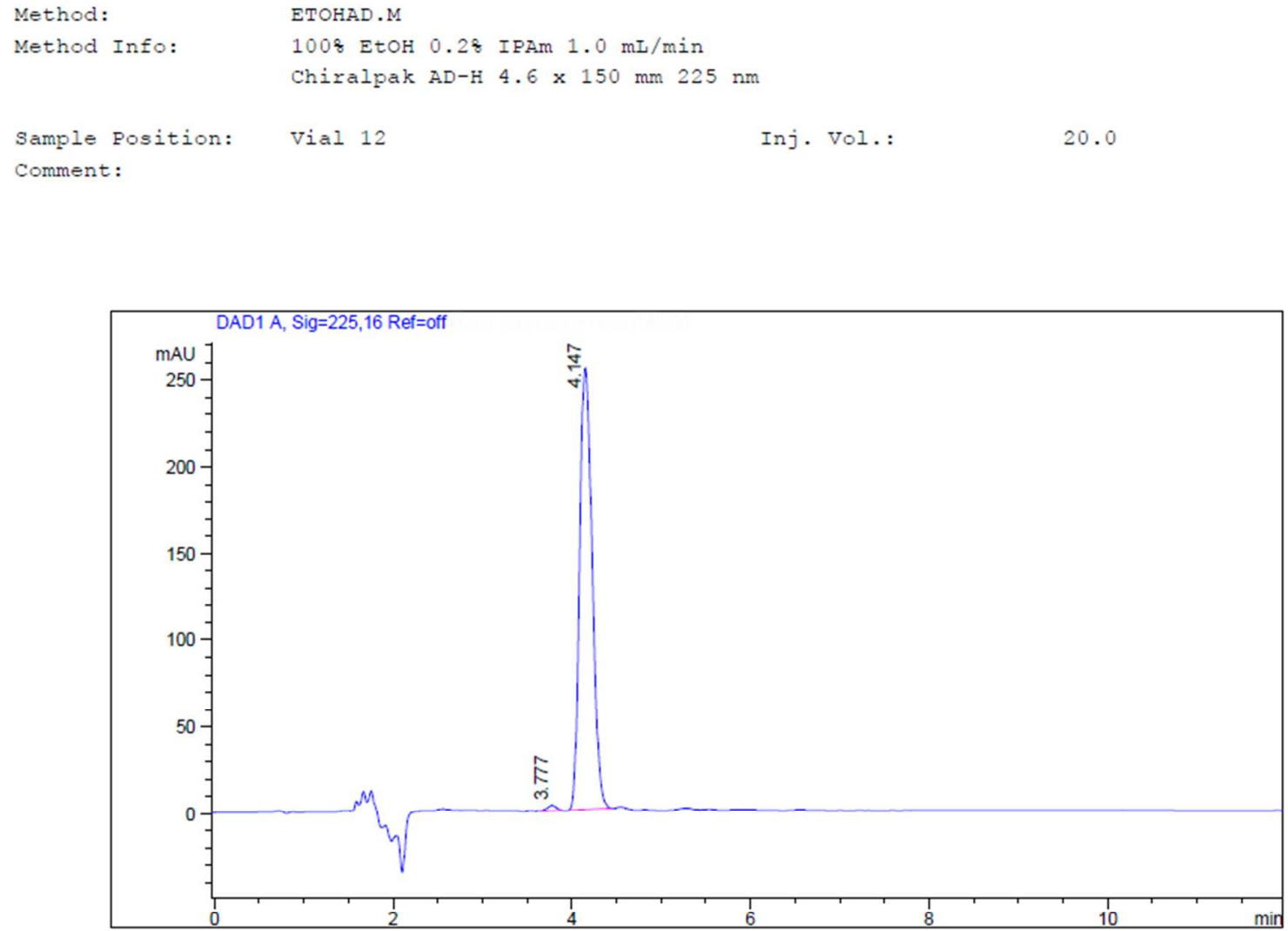

Integration results for DAD1 A, $\mathrm{Sig}=225,16$ Ref=off

Major Peak: \& Purity RT

\begin{tabular}{|c|c|c|c|}
\hline Peak RT & width & Height & \& Area \\
\hline & & - & 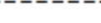 \\
\hline 3.78 & 0.121 & 3.1 & 0.5 \\
\hline 4.15 & 0.162 & 254.5 & 99.5 \\
\hline
\end{tabular}

Chiral HPLC analysis of a racemic sample of

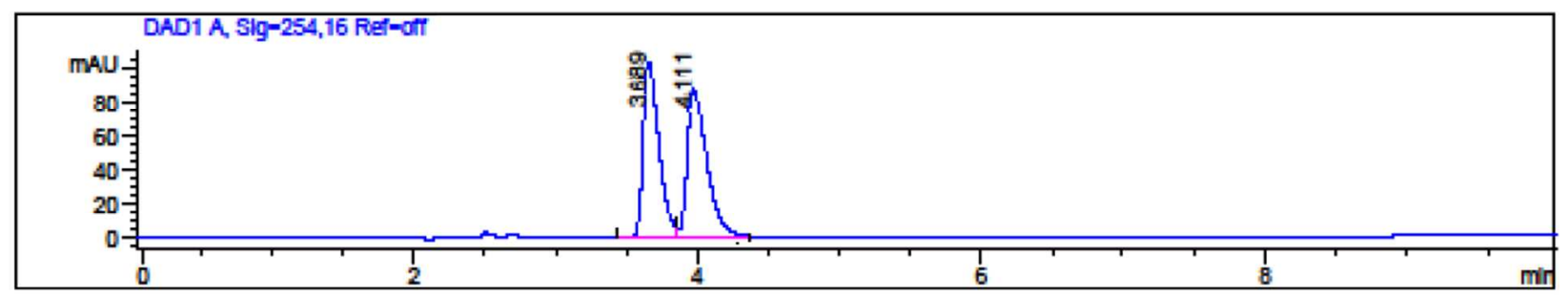

Area to Table of Signal DAD1 A, Sig=254,16

RET -

$\begin{array}{ccrr} & \text { Height } & \text { Area } & \text { Areat } \\ 3.689 & 94 & 799 & 48.21 \\ 4.111 & 81 & 859 & 51.79 \\ & & & 100.00\end{array}$




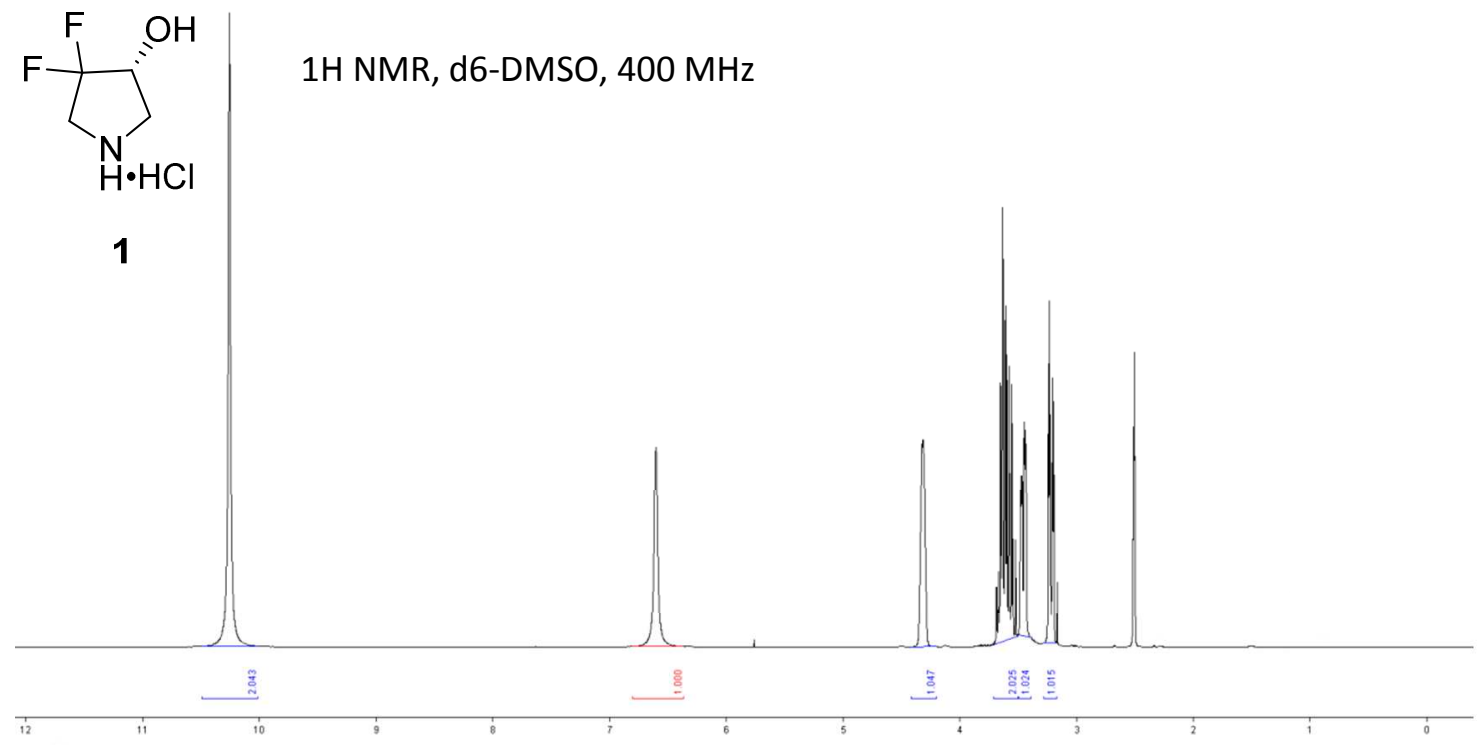

19F, d6-DMSO, $377 \mathrm{MHz}$

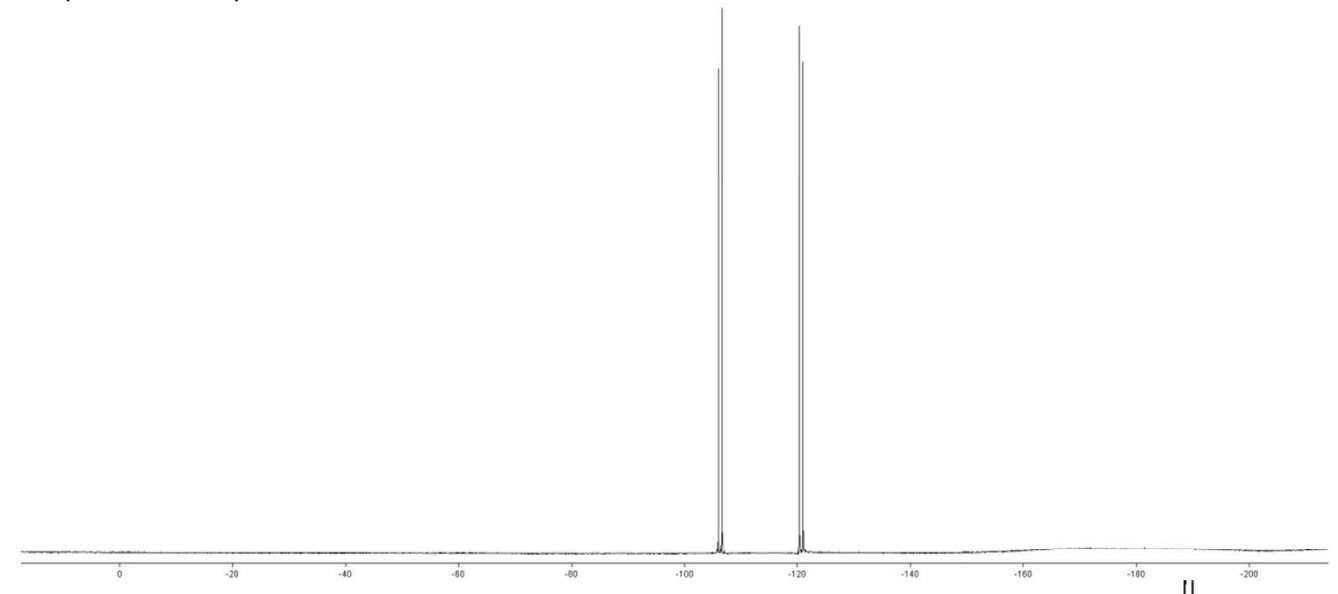

13C NMR, d6-DMSO, $100 \mathrm{MHz}$

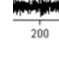




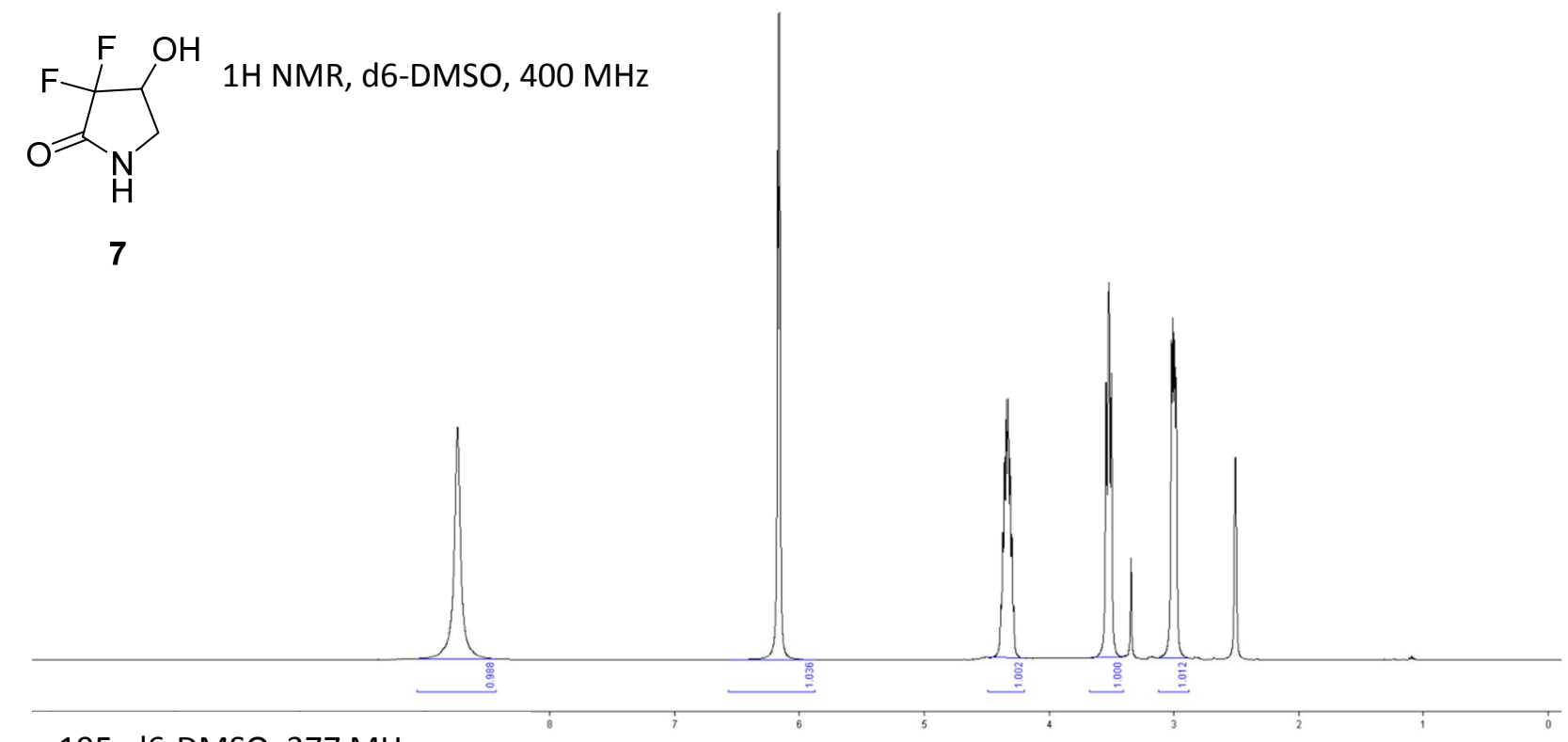

19F, d6-DMSO, $377 \mathrm{MHz}$

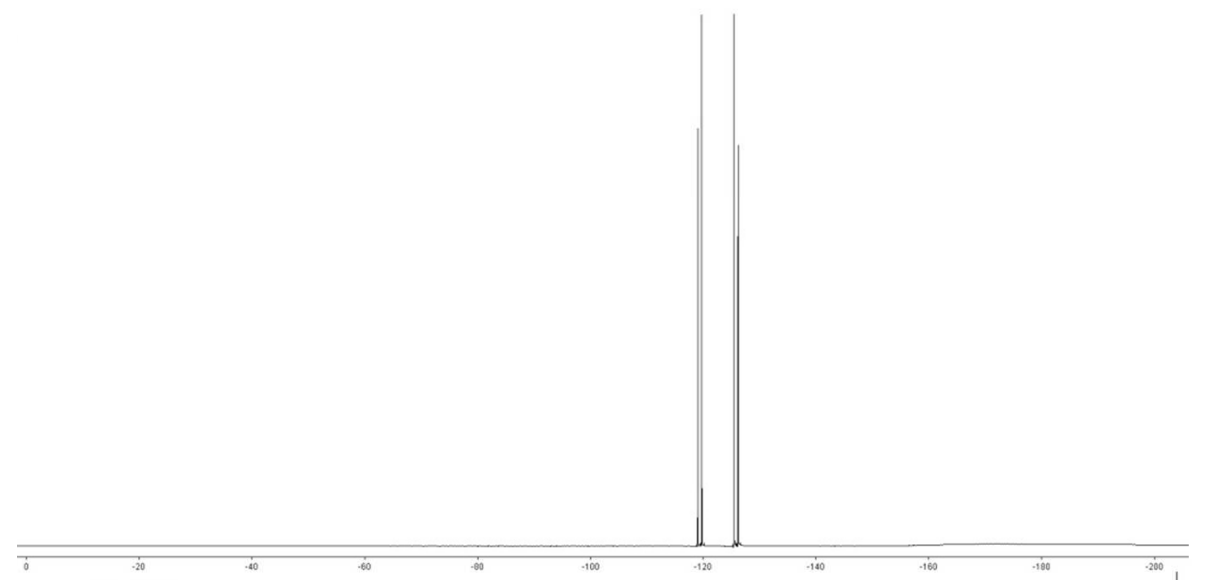

13C NMR, d6-DMSO, $100 \mathrm{MHz}$

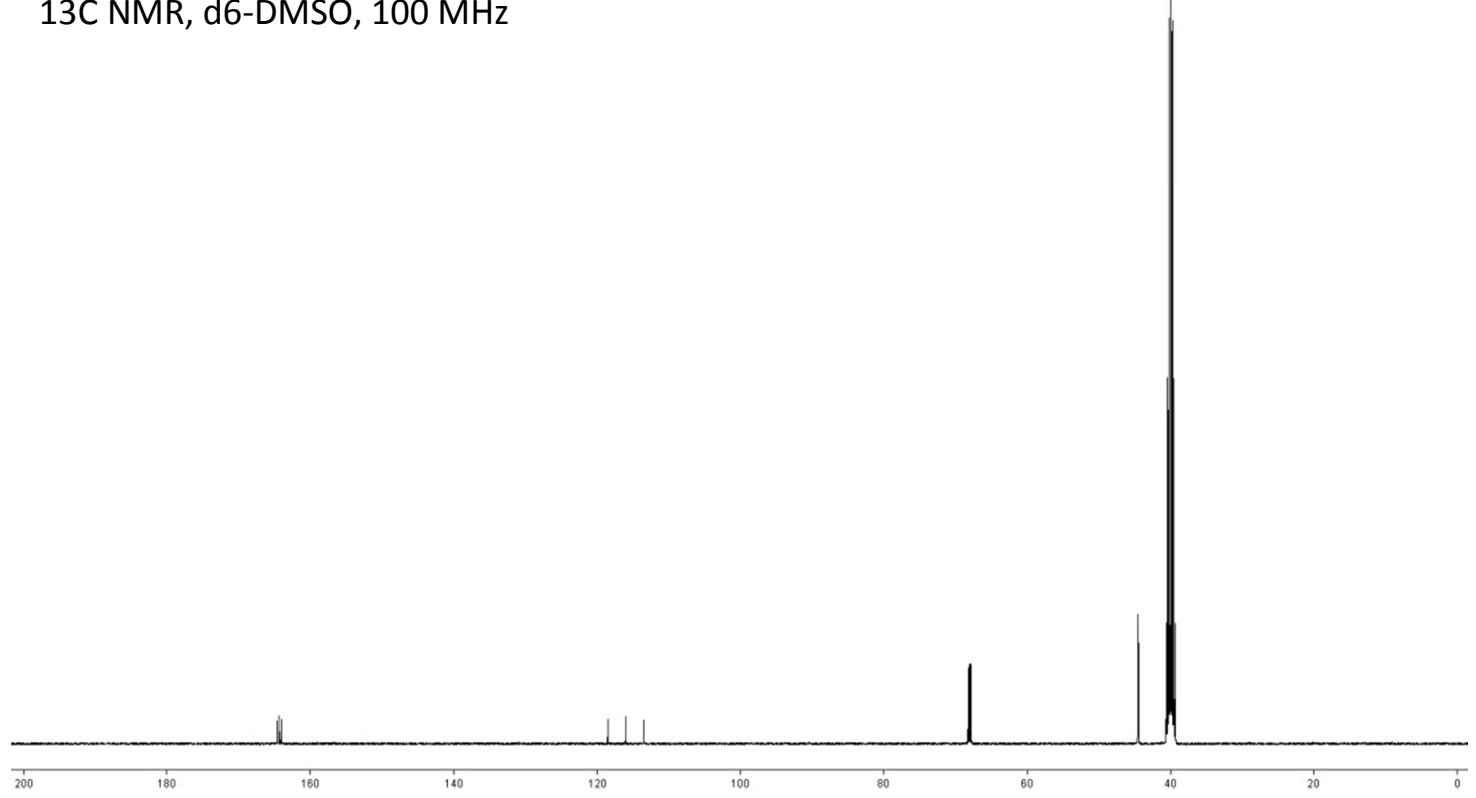




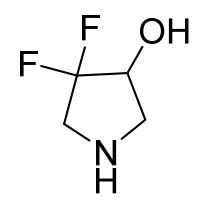

8
1H NMR, $\mathrm{CDCl}_{3}, 400 \mathrm{MHz}$

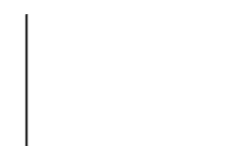

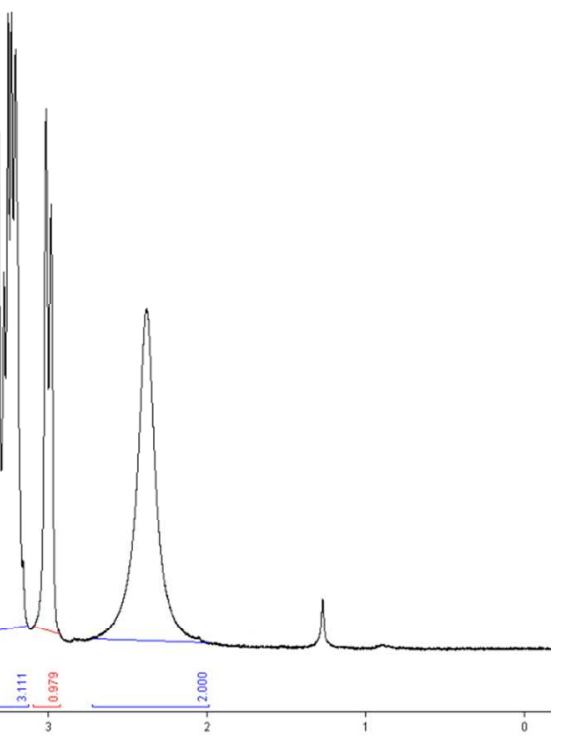

19F, $\mathrm{CDCl}_{3}, 377 \mathrm{MHz}$

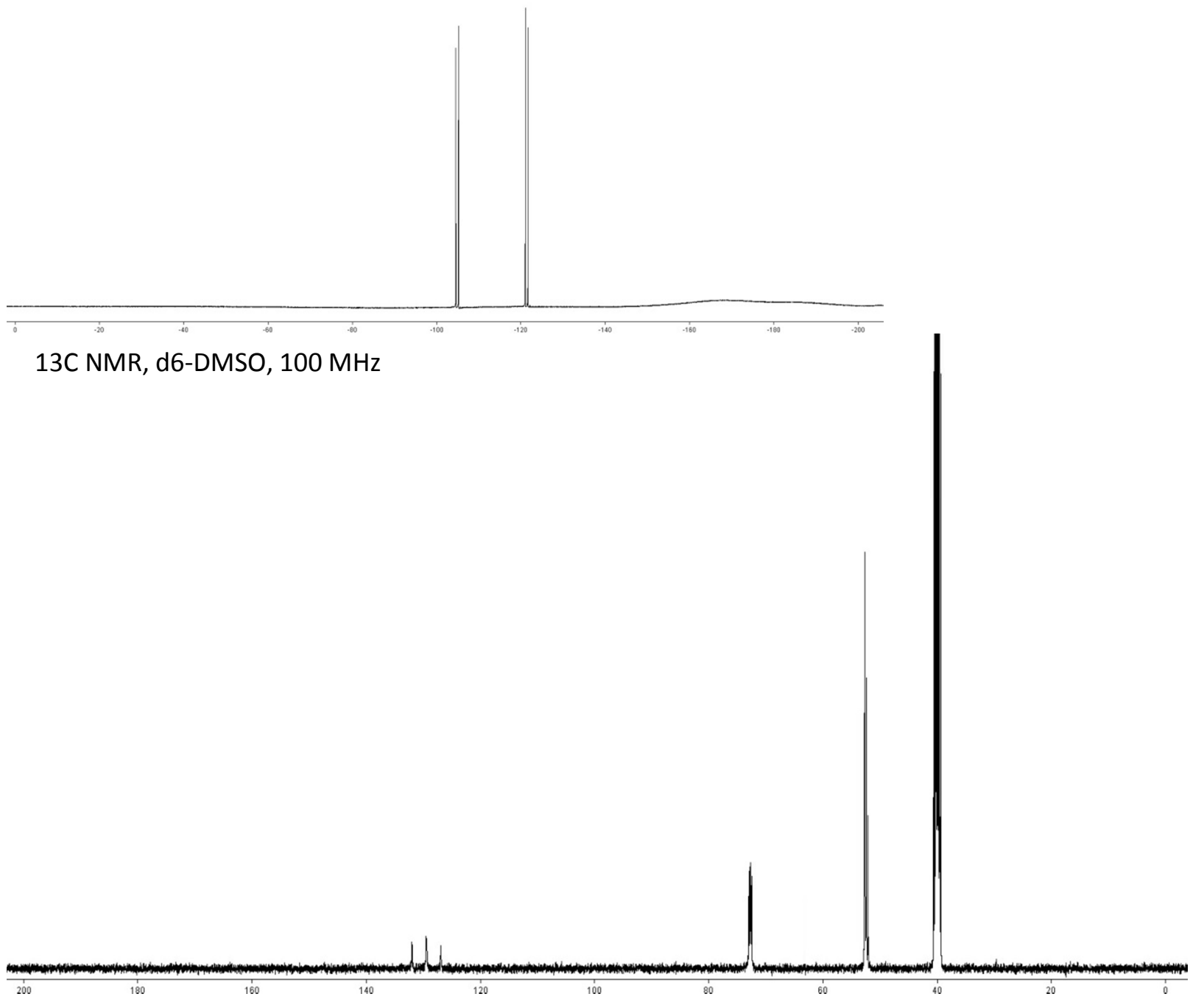

S8 


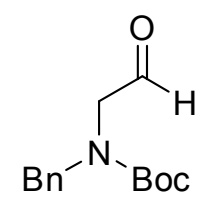

10

1H NMR, $\mathrm{CDCl}_{3}, 400 \mathrm{MHz}$

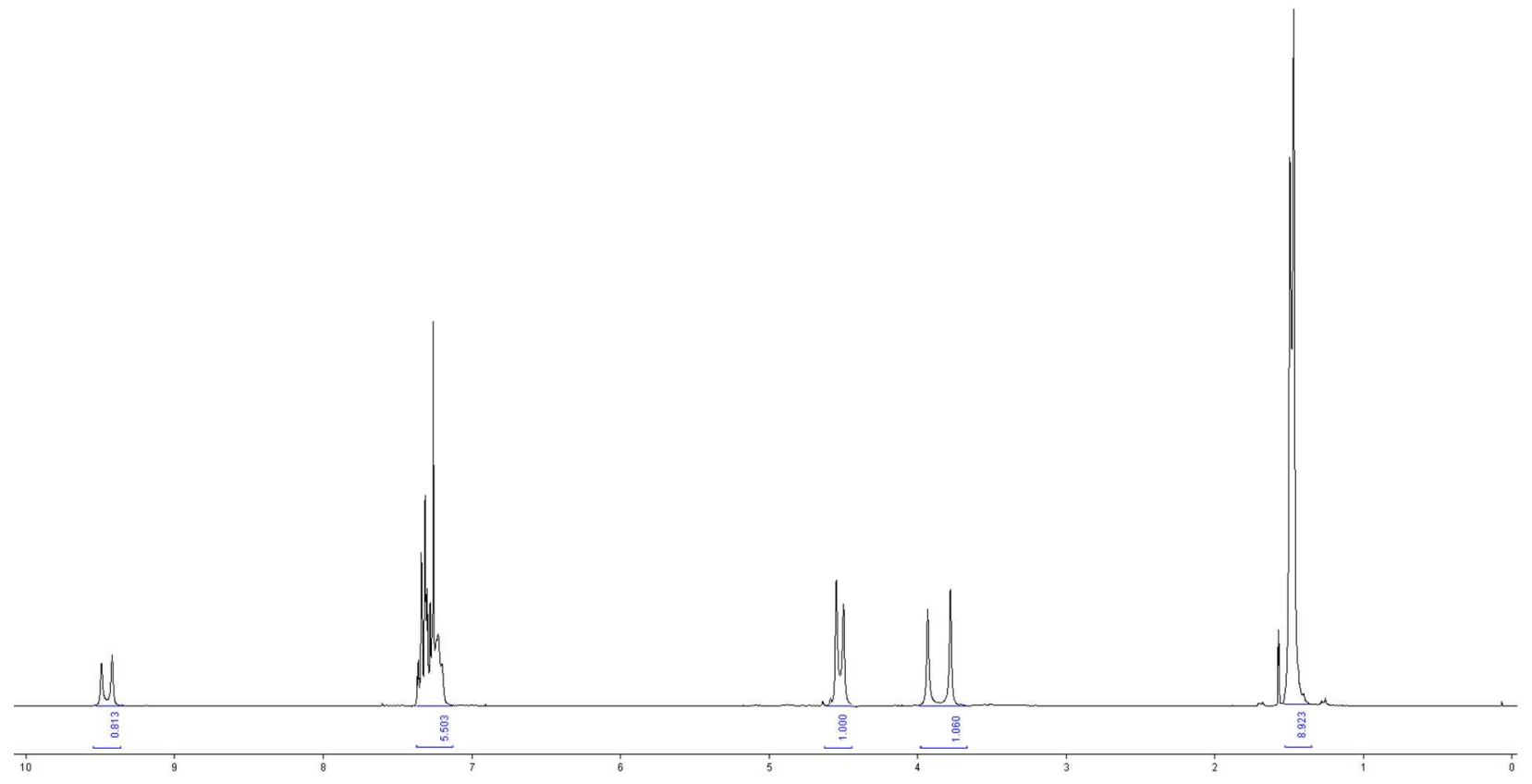

13C NMR, $\mathrm{CDCl}_{3}, 75 \mathrm{MHz}$

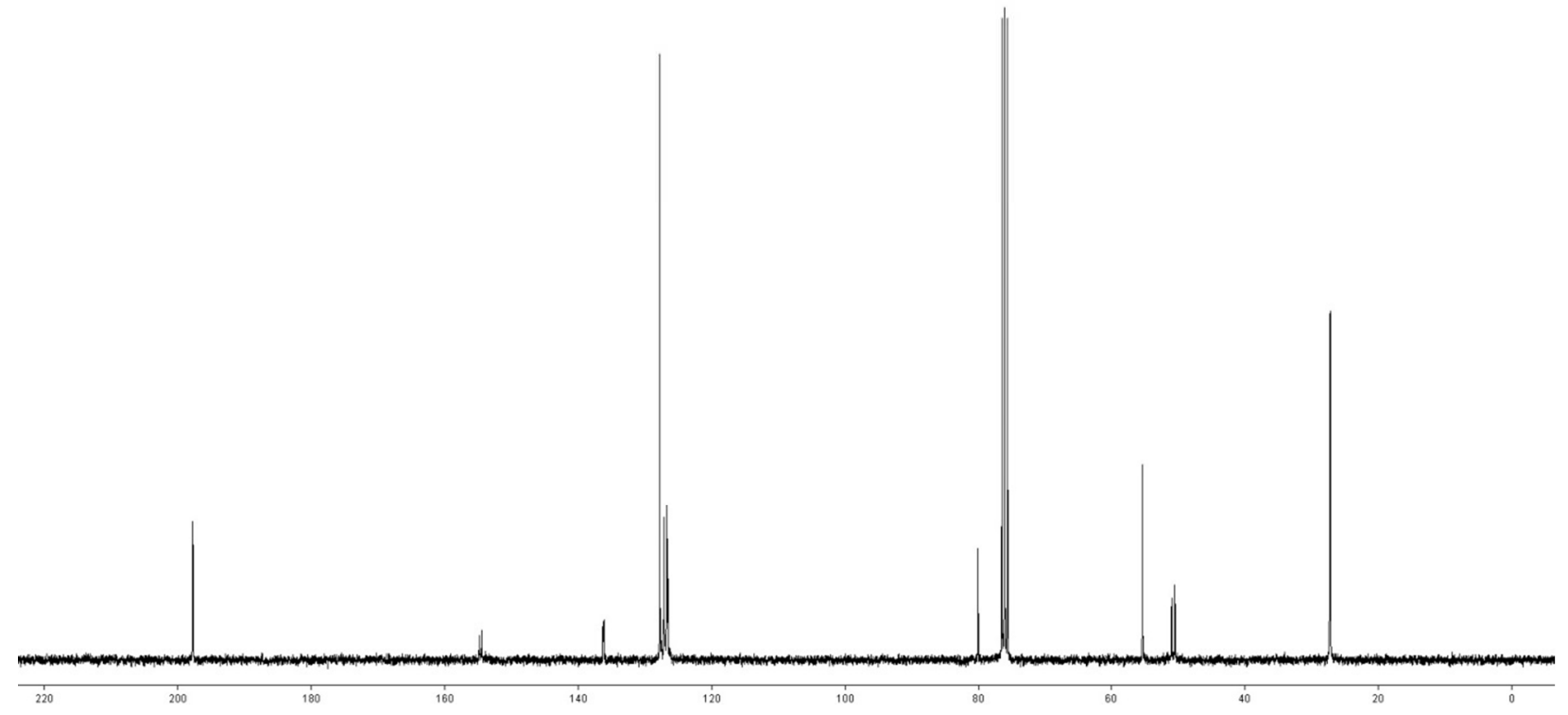




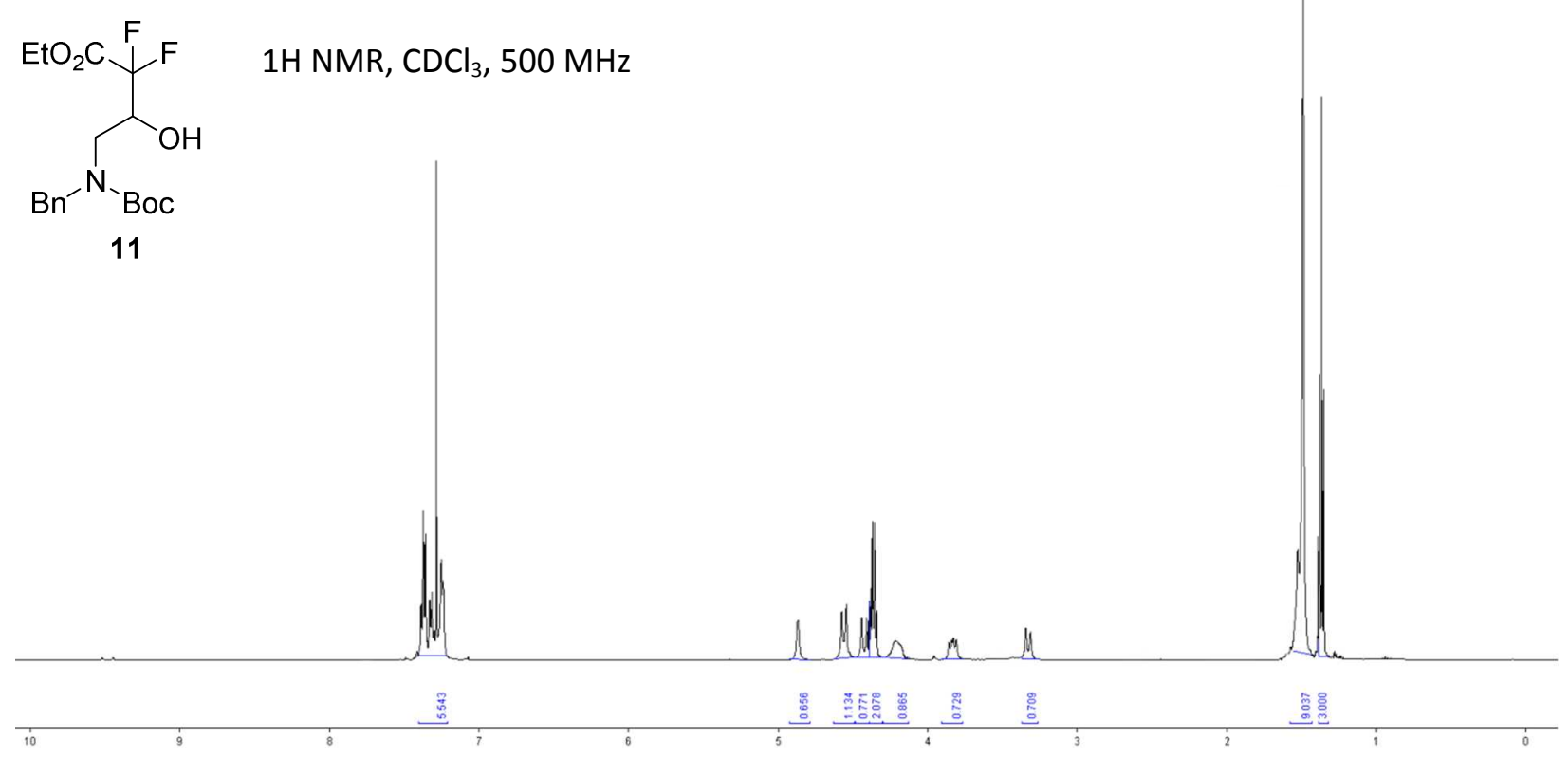

19F, $\mathrm{CDCl}_{3}, 470 \mathrm{MHz}$

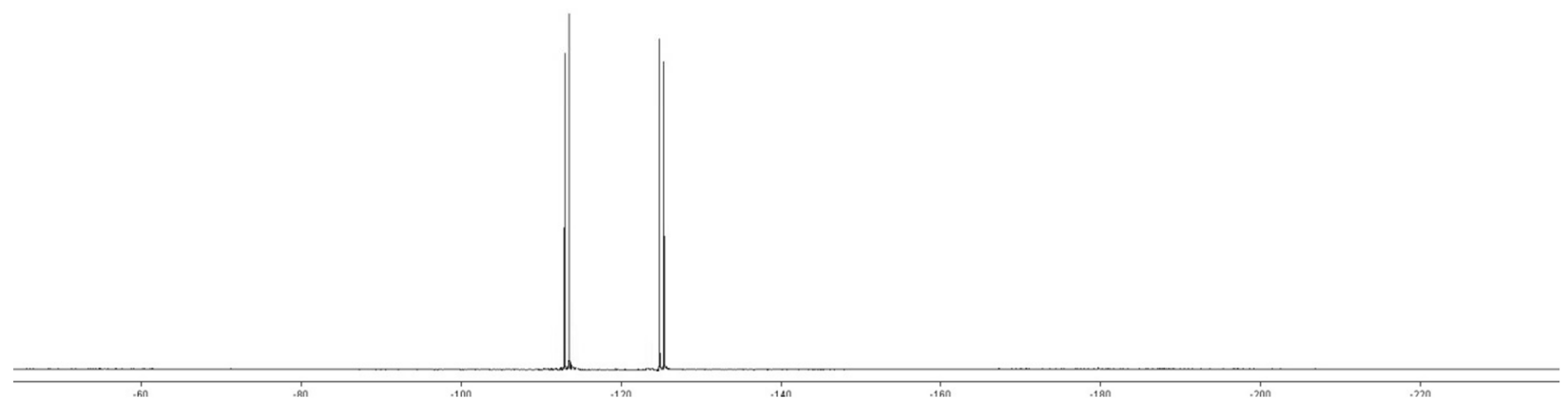

13C NMR, $\mathrm{CDCl}_{3}, 75 \mathrm{MHz}$

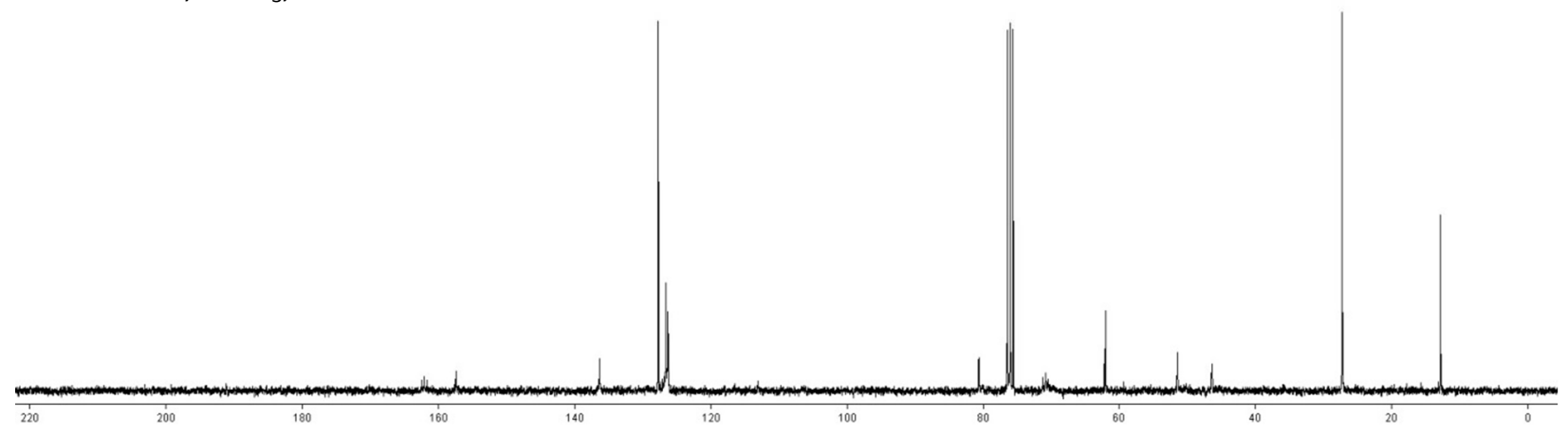




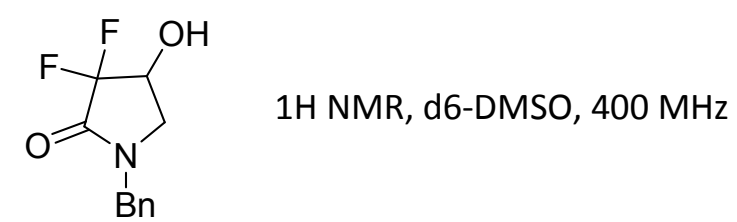

12

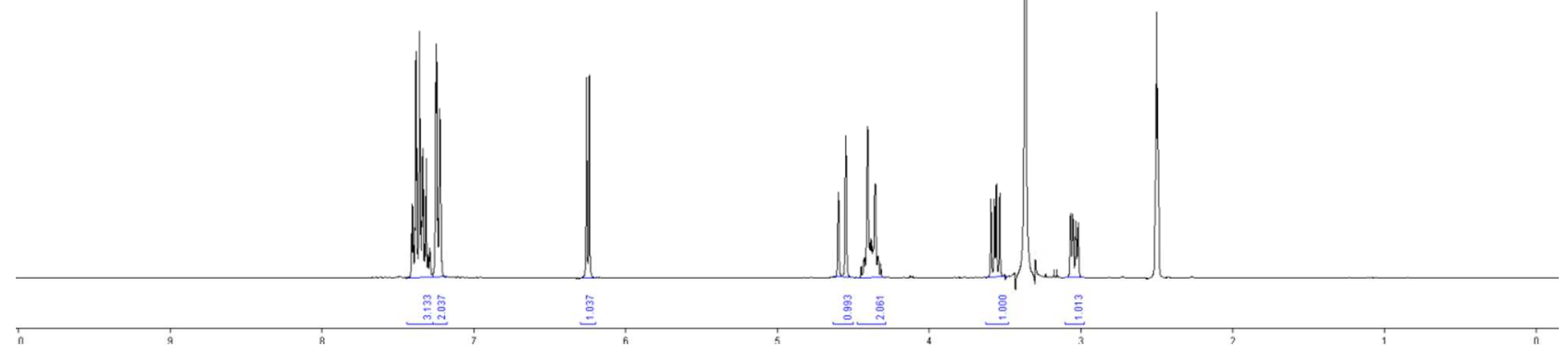

19F, d6-DMSO, $470 \mathrm{MHz}$

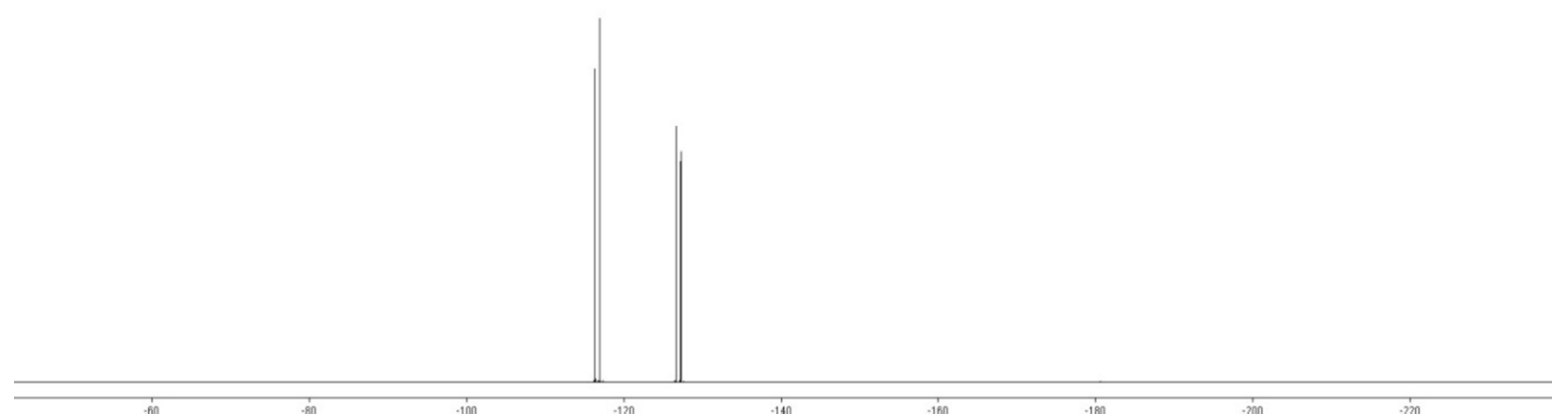

13C NMR, d6-DMSO, $75 \mathrm{MHz}$

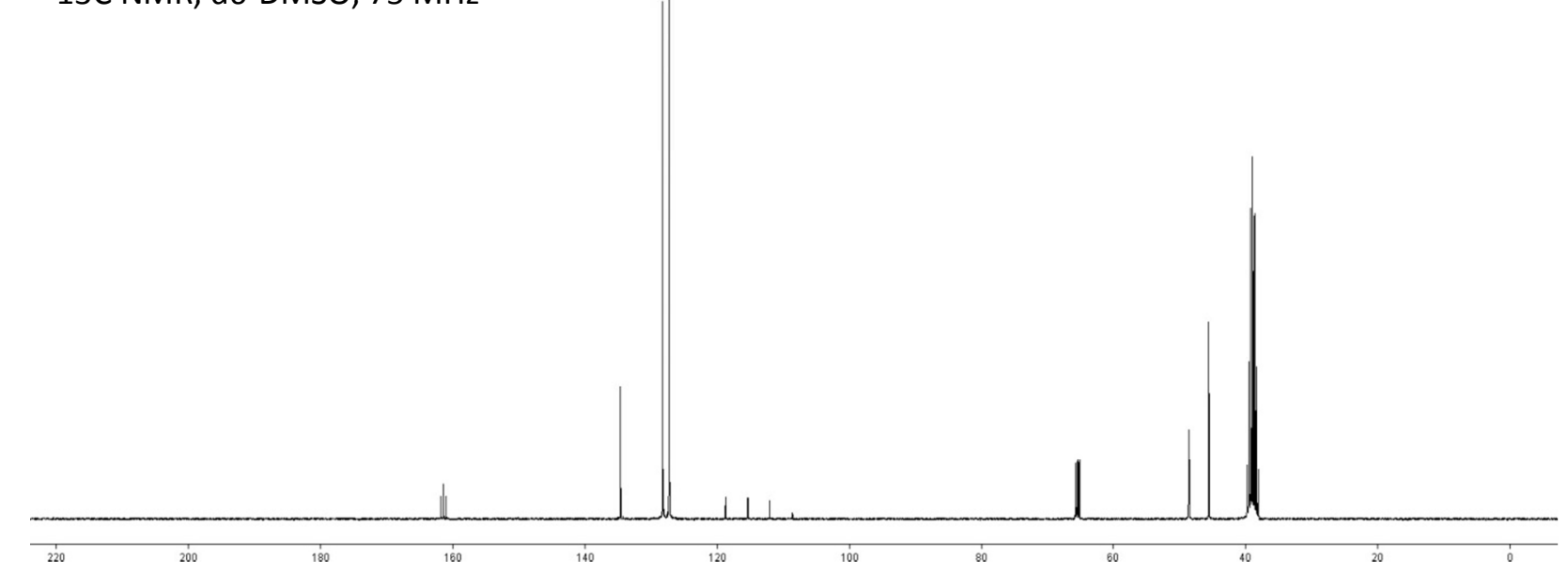




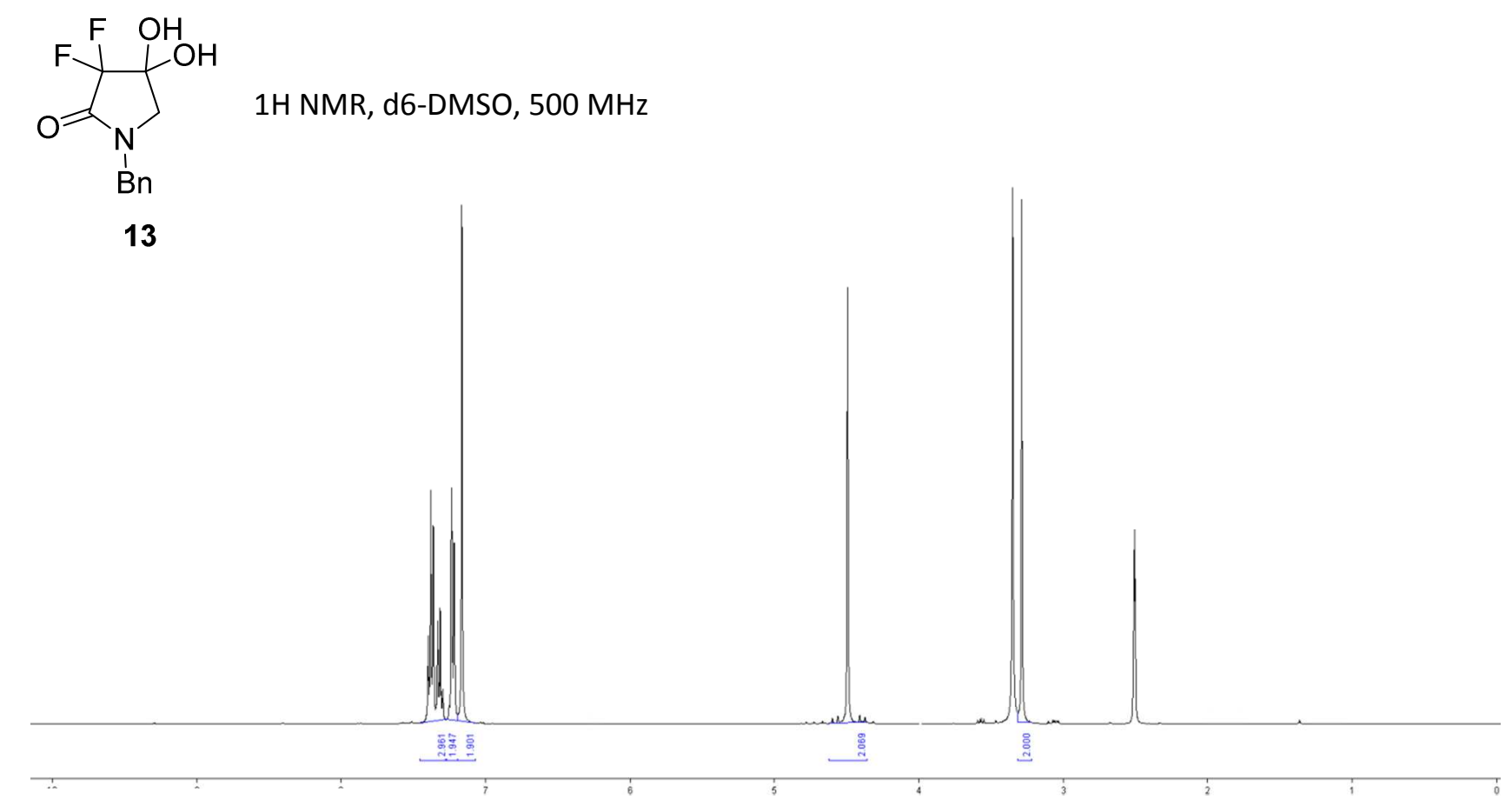

19F, d6-DMSO, $470 \mathrm{MHz}$

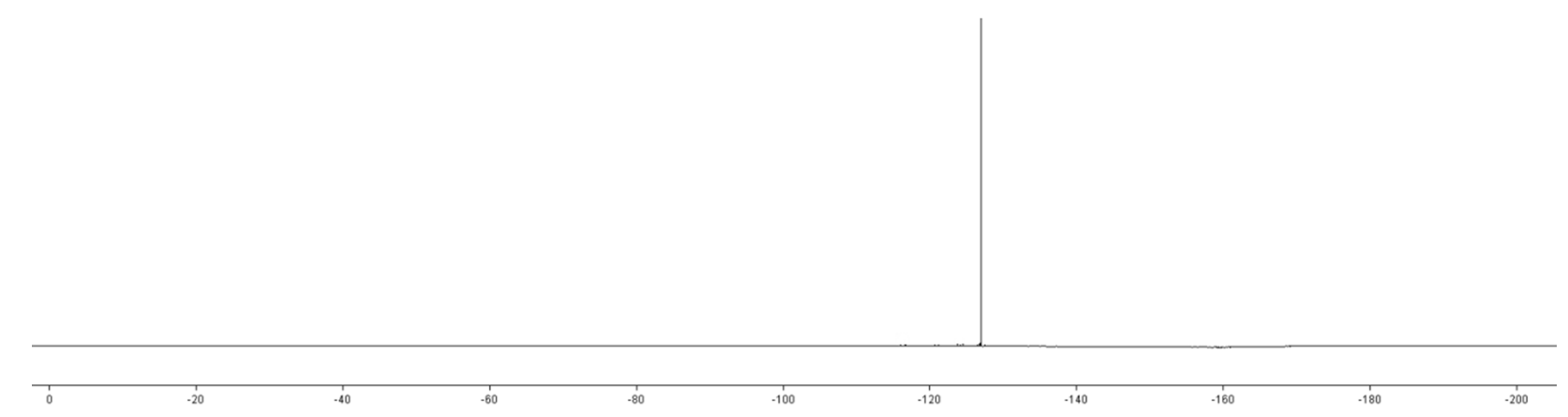

13C NMR, d6-DMSO, $75 \mathrm{MHz}$

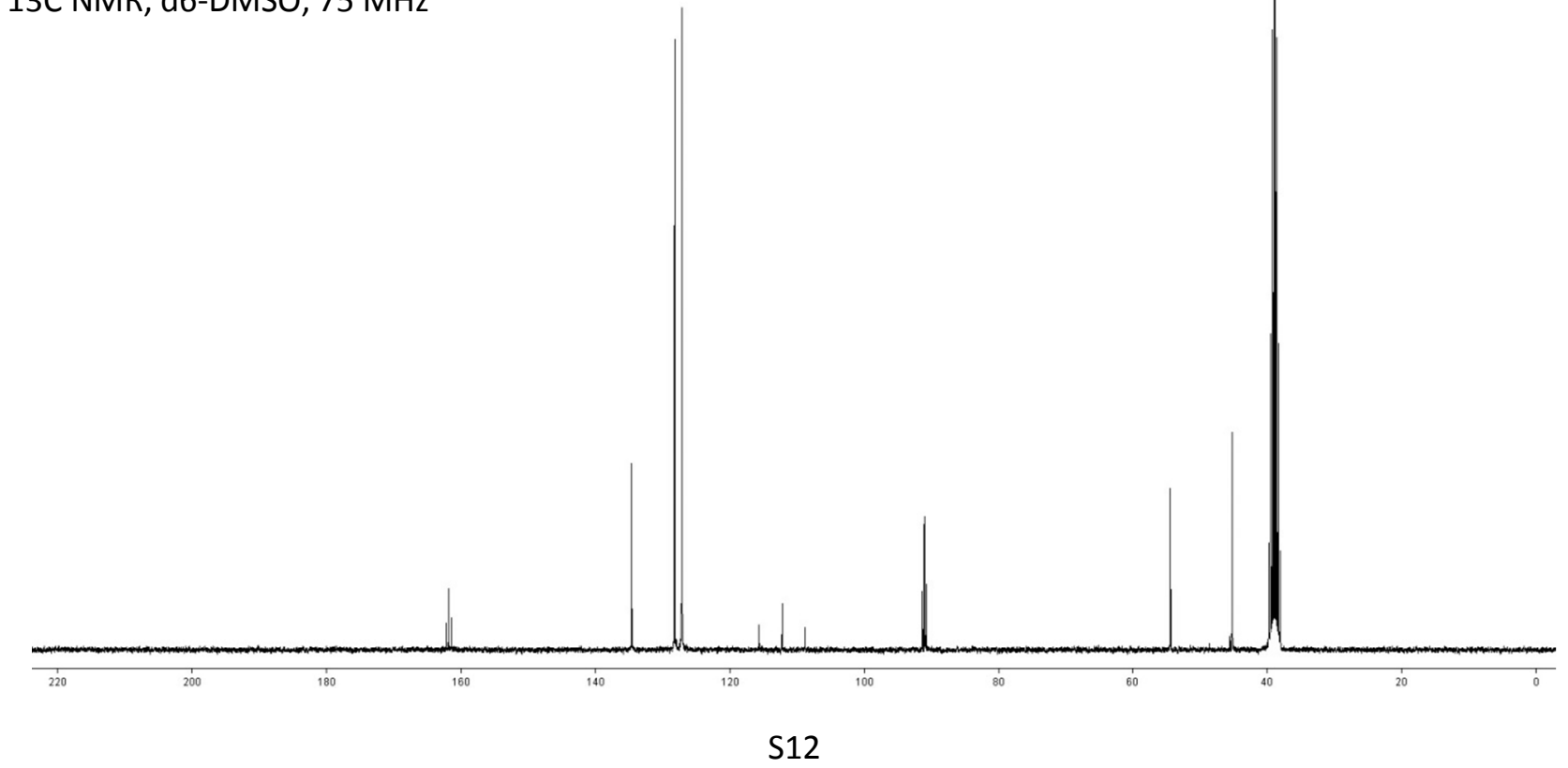




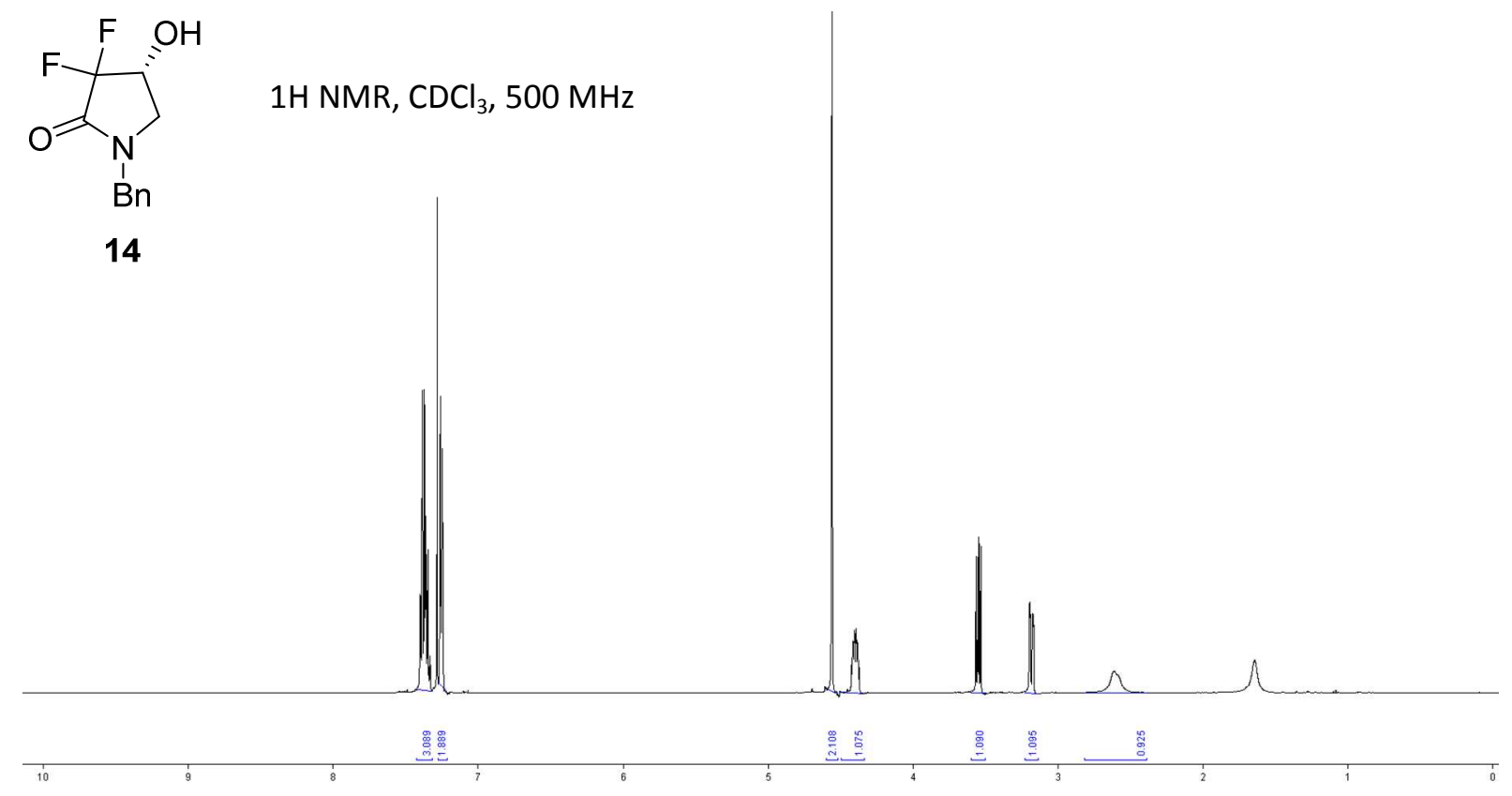

19F, $\mathrm{CDCl}_{3}, 470 \mathrm{MHz}$

13C NMR, $\mathrm{CDCl}_{3}, 75 \mathrm{MHz}$

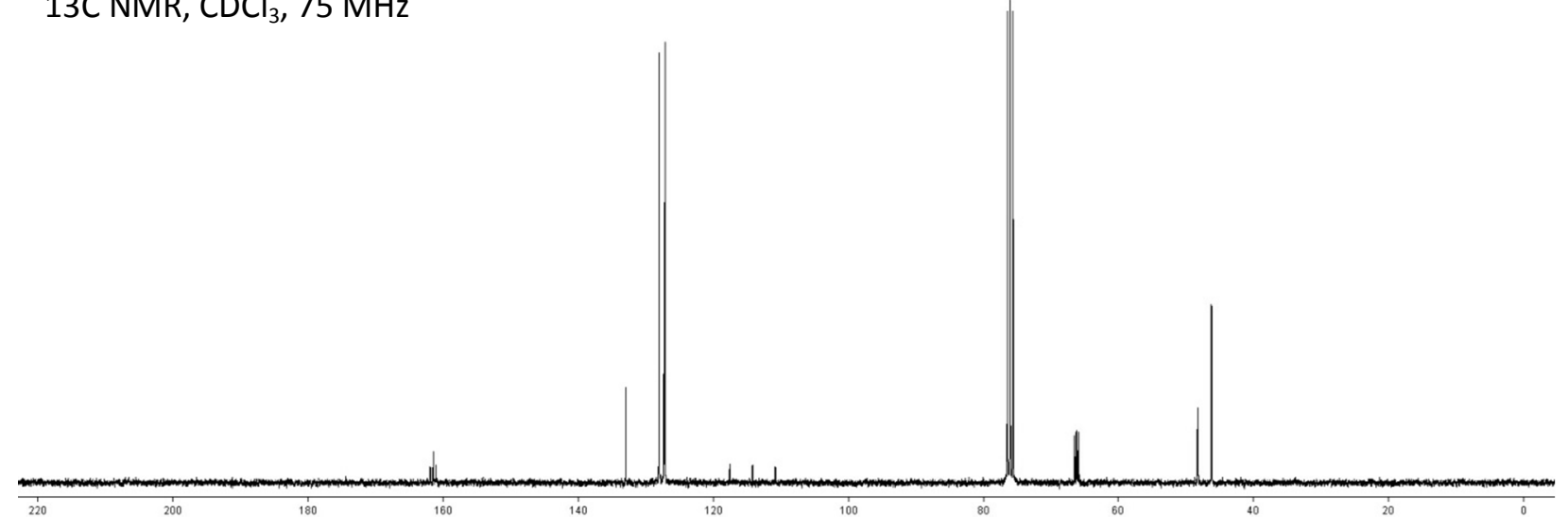




\section{Chiral HPLC analysis of compound $\mathbf{1 4}$}

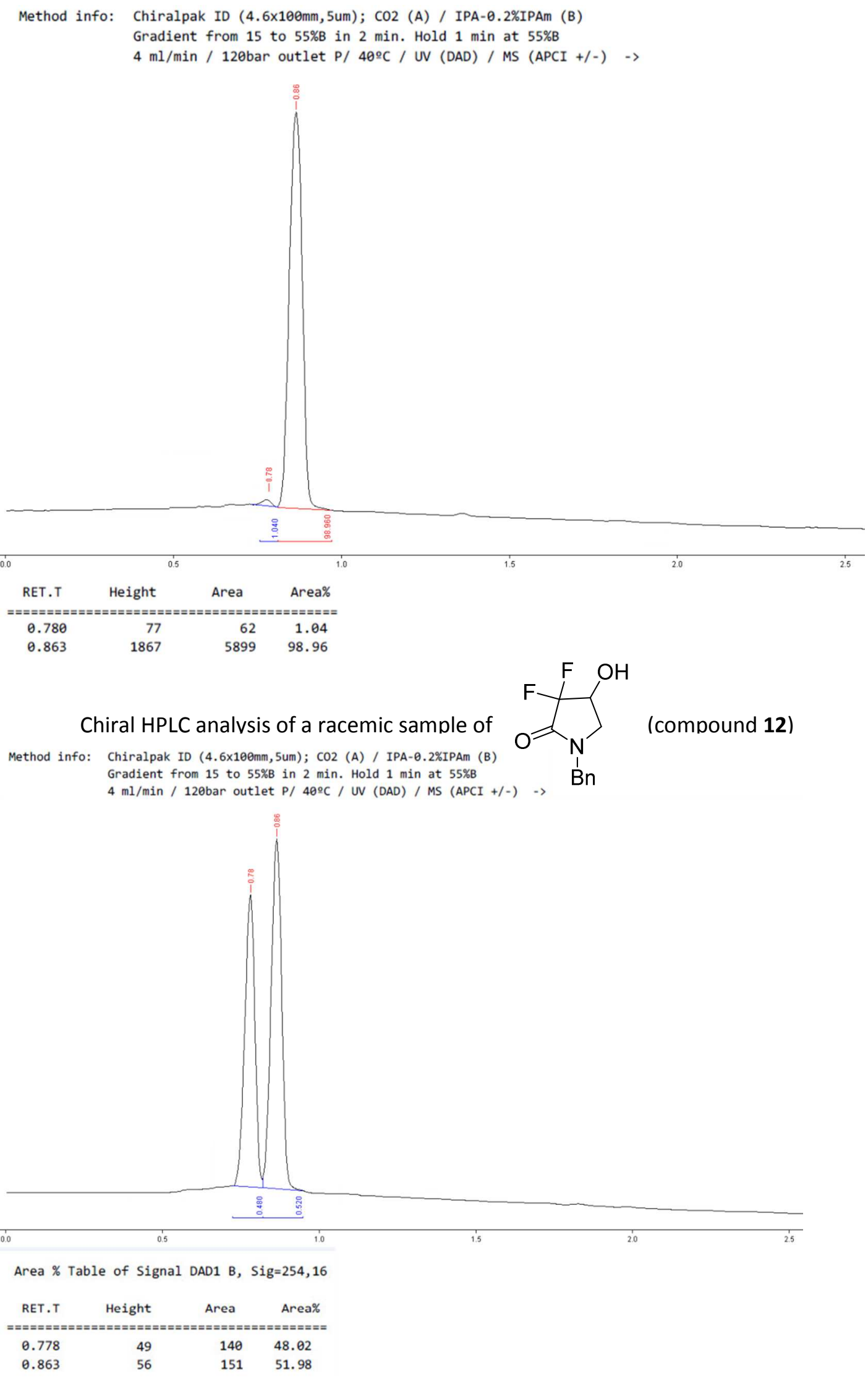




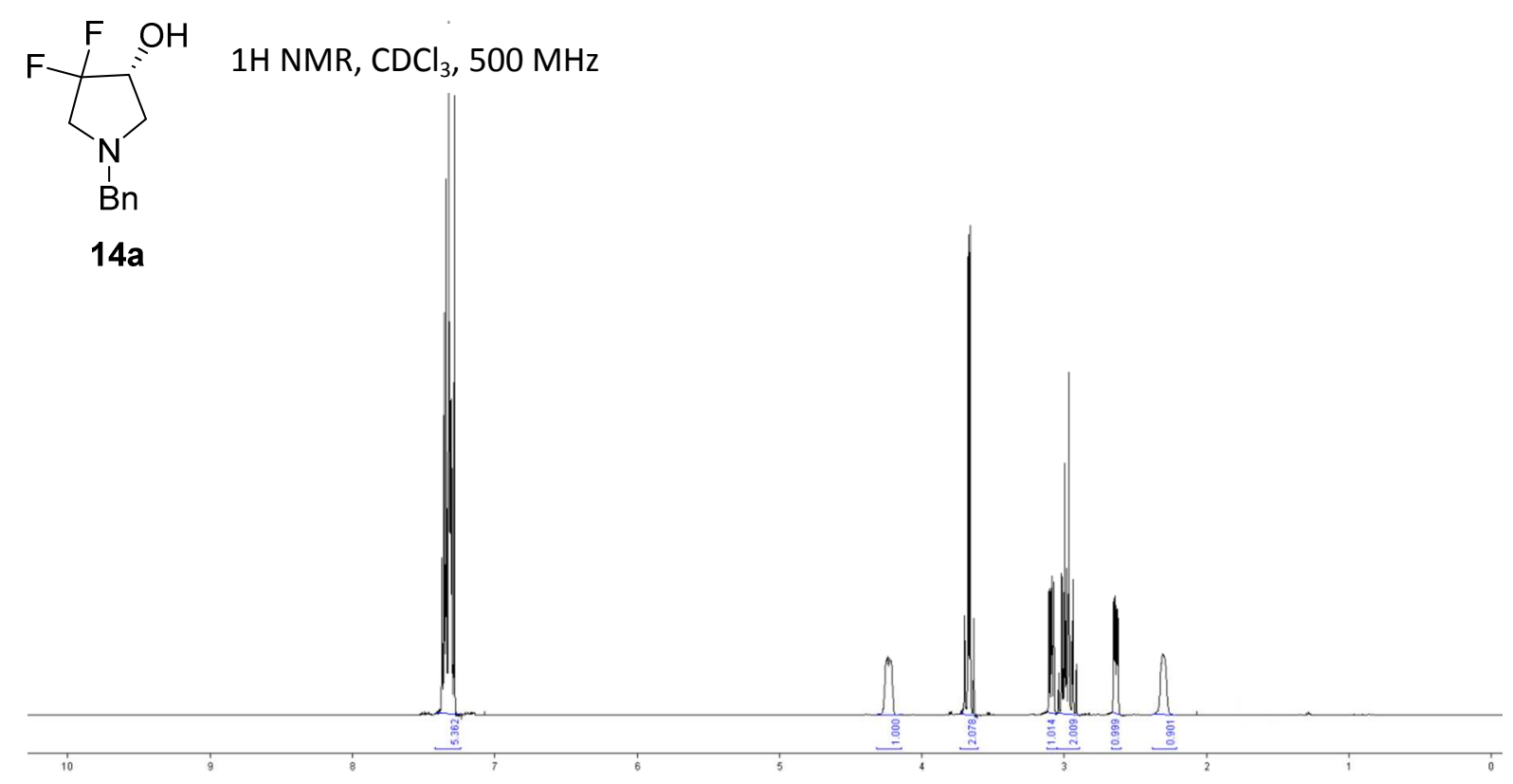

$19 \mathrm{~F}, \mathrm{CDCl}_{3}, 470 \mathrm{MHz}$

13C NMR, $\mathrm{CDCl}_{3}, 75 \mathrm{MHz}$

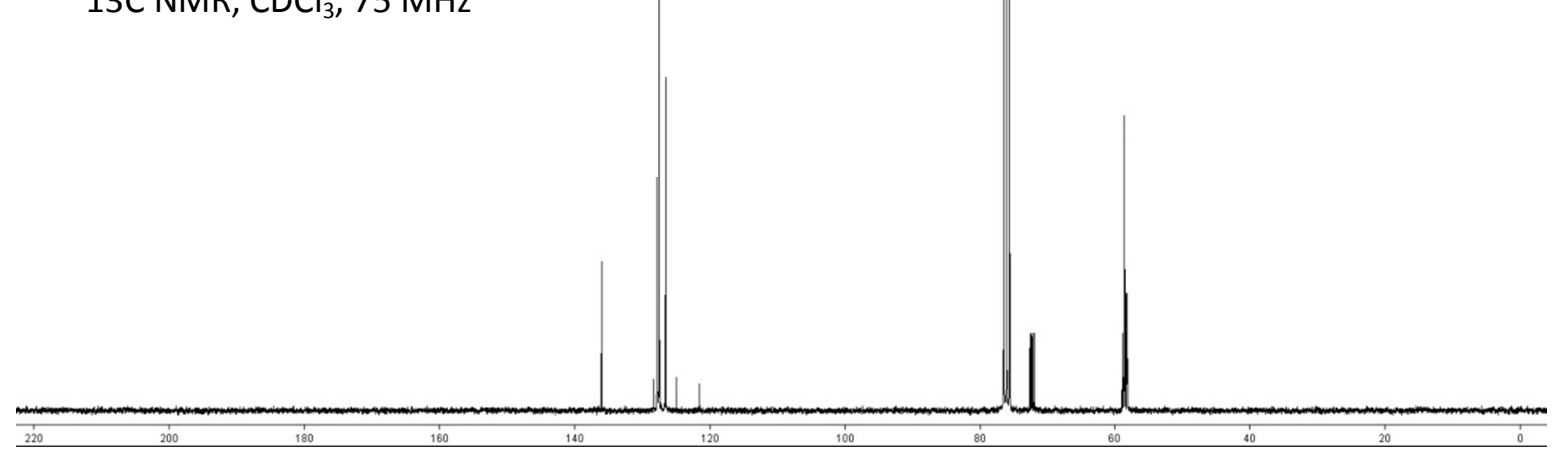

